\title{
Expression of emotion through musical parameters in 3-and 5-year-olds
}

\section{Saarikallio, Suvi}

2019-10-20

Saarikallio , S , Tervaniemi , M , Yrtti , A \& Huotilainen , M 2019 , ' Expression of emotion through musical parameters in 3-and 5-year-olds ', Music Education Research , vol. 21 , no. 5 , pp. 596-605 . https://doi.org/10.1080/14613808.2019.1670150

http://hdl.handle.net/10138/327961

https://doi.org/10.1080/14613808.2019.1670150

acceptedVersion

Downloaded from Helda, University of Helsinki institutional repository.

This is an electronic reprint of the original article.

This reprint may differ from the original in pagination and typographic detail.

Please cite the original version. 


\title{
Expression of emotion through musical parameters in 3- and 5-year- olds
}

\author{
*Suvi Saarikallio, \#Mari Tervaniemi, *Antti Yrtti, \& \#Minna Huotilainen \\ *Department of Music, Art and Culture Studies, Finnish Centre for Interdisciplinary \\ Music Research University of Jyväskylä, Finland
}

\#CICERO Learning and Cognitive Brain Research Unit, Faculty of Education, University of Helsinki, Helsinki, Finland

\section{Corresponding author}

Suvi Saarikallio, suvi.saarikallio@jyu.fi,_358 505361900

University of Jyväskylä, Department of Music, Art and Culture, Studies P.O. Box 35

FI-40014 University of Jyväskylä

\section{Short biographical notes on contributors}

Dr. Suvi Saarikallio is a Docent of Music Psychology, working as Senior Researcher and Department Vice-Head at the Department of Music, Art and Culture Studies, University of Jyväskylä. Saarikallio specializes in music, emotions, and psychosocial development.

Prof. Mari Tervaniemi is Head of the CICERO Learning, working at the Cognitive Brain Research Unit and Faculty of Educational Sciences, University of Helsinki. Tervaniemi specializes in mechanisms of human auditory cognition, emotion, and expertise.

MA Antti Yrtti conducted his Master's thesis at the University of Jyväskylä as part of this research project and currently works as an Audio Engineer at Storytel AB.

Prof. Minna Huotilainen works as Professor of Education at the Faculty of Education and at the Cognitive Brain Research Unit, University of Helsinki. Huotilainen specializes in neural mechanisms of learning and cognition. 


\section{Expression of emotion through musical parameters in 3- and 5-year- olds}

While use of musical parameters for emotional expression has been extensively studied, little is known about which specific musical parameters children at different ages are able to use for expressing specific emotions. We used a novel interface called Music Box that allows modification of musical parameters while music is being played in real time. Children ( $N=37,18$ girls) at the age of 3 and 5 were asked to modify three parameters - tempo, loudness, and pitch - in expressing three emotions - happiness, sadness, and anger. We hypothesized that both 5-year-olds and 3-year-olds could use each of the parameters in differentiating between the emotions. Results showed that 3-year-olds were equally competent than 5-yearl-olds in using the parameters. Differences based on gender and music background were additionally measured. The study provides knowledge on normative development of children's ability for musical expression of emotion, which can be used to inform music education and music therapy practices.

Keywords: emotional expression; children; basic emotions; musical parameters 


\section{Bakcground}

It is often seen that musical expression can convey basic emotions (e.g., Gabrielsson \& Lindström, 2001; 2010; Juslin \& Laukka, 2003; 2004). The current study examined the development of this ability in childhood, with the general aims of advancing understanding of children's emotional development and providing basic background knowledge for educational and therapeutic use of music with children.

\subsection{Prior research on music as expression of emotion mostly focuses on adults}

A commonly studied aspect of emotional communication through music has been the set of musical features (e.g., tempo, sound level, timbre) that can be associated with the performers' expression (Gabrielsson \& Lindström, 2001; Juslin \& Timmers, 2010) and the listeners' perception (Juslin \& Laukka, 2004) of discrete emotions such as happiness, sadness, anger, fear, and tenderness. Extensive reviews of musical features that are observed to relate to either intended or perceived expression of basic emotions (Gabrielsson \& Lindström, 2010; Juslin \& Timmers, 2010; Juslin \& Laukka, 2004) show that happiness is typically characterized by fast tempo, major mode, medium-high or high sound level, high pitch, staccato articulation, and bright timbre while sadness is typically related to slow tempo, minor mode, low sound level, low pitch, legato articulation, and dull timbre. Anger is characterized by fast tempo, high sound level, high pitch, staccato articulation, and sharp timbre and spectral noise. Overall, studies show that individuals connect the abovementioned features to emotions in a relatively consistent manner, at least when differentiating basic emotions from each other (Bigand, Vieillard, Madurell, Marozeau, \& Dacquet, 2006; Juslin \& Laukka, 2004; Juslin \& Timmers, 2010; Peretz, Gagnon, \& Bouchard, 1998; Vieillard, Peretz, 
Gosselin, Khalfa, Gagnon \& Bouchard 2008). Differences between musically trained and untrained individuals also appear relatively small (Bigand et al, 2006; Fredrickson, 2000; Juslin, 1997). However, most research on emotion expression and recognition in music has been conducted with adult samples and much less is known about the development of this behavior during childhood.

\subsection{Children can distinguish basic emotions in music}

The general childhood emotional development provides an overall context for understanding the musical expression of emotion by children. Regarding general emotional development, 3-year-old children are able to express almost as wide a range of emotions as adults and also capable of evaluating their emotional behavior (Lewis, 2000). However, children's insight into the emotions of others significantly develops during the preschool years (2-5years) (Saarni, 1999), serving as a foundation for prosocial emotional communication and empathy. Their ability to perceive emotional content further develops during the early school age, and particularly their understanding of simultaneously presented emotions is not at adults' level (Schubert \& McPherson, 2006). Findings regarding music-related emotion perception are somewhat in line with the general development and demonstrate children as relatively competent in perceiving basic emotions in music. For instance, 3-5-year olds give highly similar responses to adults when asked to match musical pieces to facial expressions reflective of basic emotions (happy, sad, angry, fear and neutral), (Nawrot, 2003), and 5-yearolds are able to separate positive and negative expression in classical music excerpts (Cunningham \& Sterling, 1988; Terwogt \& van Grinsven, 1991). Perception of happiness and sadness in music seem to precede other emotions: 3-year-olds distinguish happy and sad music (Kastner \& Crowder, 1990) while 4-6-year olds correctly identify happiness, sadness, anger and fear in music (Dolgin \& Adelson, 1990; Cunningham \& 
Sterling, 1988). Children quite easily confuse fearful and angry displays (e.g., Boone \& Cunningham, 2001; Thompson, Schellenberg, \& Husain, 2004; Robazza et al., 1994; Terwogt \& van Grinsven, 1991), and sometimes angry and happy expressions (Stachó, 2013).

\subsection{Differently aged children may use different musical parameters in emotion judgments}

While the abovementioned studies generally evidence children as capable in differentiating basic emotions in musical expression, they do not provide conclusive understanding of age differences, particularly in terms of which musical parameters children use to make their emotion judgments. Different studies target children at different ages, and reliable comparison between studies is difficult due to varying musical stimulus materials. Many studies do not control for the specific musical parameters that children use for their emotion recognition judgments or the parameters differ between the studies. For instance, the abovementioned study of Stachó et.al (2013) modulated only performance features (e.g., articulation, tempo, microtiming, sound level), while the compositional features (e.g. mode, melody, harmonic and rhythmic structure) were kept constant.

One distinction of musical parameters relates to cultural learning. Some parameters reflect psychophysical aspects of emotional expression - being comparable to features of speech prosody - and can be considered to be more universal, while other parameters relate to more symbolic and culturally learned aspects of emotional expression (Balkwill \& Thompson, 1999). Tempo, for instance, is linked to arousal in music across cultures, while the connections of happiness to major mode and sadness to minor mode are particularly typical to western music (Schubert \& McPherson, 2006; Trehub, Hannon, \& Schachner, 2010). From this, it has been proposed that some 
performance cues, such as tempo and loudness, are connected with emotion in a relatively innate way and thus be detected by younger children than the features dependent on cultural learning (Schubert \& McPherson, 2006; Trehub et al. 2010). This has also been confirmed with empirical data. Dalla Bella et al. (2001) modified both tempo and mode of pre-existing classical music pieces, and reported that 3-4-year-olds failed to distinguish happy and sad expression, 5-year-olds' responses were affected by tempo change only, and 6-8-year-olds and adults were affected by both mode and tempo manipulations. Similarly, Gregory, Worrall and Sarge (1996) showed that 7-8 year-olds but not 3-4-year-olds were able to distinguish between sad and happy expression when only mode of the excerpts was modified. Based on studies so far, it appears that the culturally based tonal understanding becomes established only between 6-9 years of age (Dalla Bella et al., 2001; Gregory et al. 1996; Krumhans1 \& Keil, 1982; Shuter-Dyson \& Gabriel, 1981). In addition, children seem to associate major music with happiness earlier than minor music with sadness (Cunningham \& Sterling, 1988; Dolgin \& Adelson, 1990; Gerardi \& Gerken, 1995), which may be due to greater exposure to major than minor mode, as the major mode is typical for children's music in the Western culture (Gerardi \& Gerken, 1995).

Overall, it seems that the psychophysiological features reign in the emotion judgments of children below 6 years of age. These features are actually relevant also for adults: features linked to the activation dimension, tempo and loudness in particular, appear to reign over the other features as a basis for adults' emotional judgments of music (Gabrielsson \& Lindström, 2010). This may explain tendencies to confuse anger to happiness, or to fear, as all of these emotions are relatively high on the arousal/activation dimension of emotion (Russel, 1980). Moreover, certain musical features may only be relevant for certain emotions, and there may be interactions between the features. Eerola, Friberg, and Bresin (2013), for instance, showed that 
while sound level (loudness) generally is an important factor for the perception of emotion in music, it appears to be of particularly low importance for sad and happy emotions. Webster and Weir (2004) showed that the typical positive association between tempo and happiness was inverted among minor, nonharmonized phrases, and Behrens \& Green (1993) demonstrated an interaction between an instrument and emotion: fear was most accurately perceived when the piece was performed with a violin.

Taken together, these studies show that in order to comprehensively understand children's development of emotional comprehension of music it is important not only to establish whether a child (at a given age) can identify a certain emotion in music, but also to elaborate the musical features that the child employs in making the judgments. The universal features (e.g. tempo, pitch, loudness, timbre) appear particularly relevant for small children, yet their relevance for particular emotions at particular ages is not well established.

\subsection{The need for research on children's emotion expression}

The abovementioned studies provide some overview of children's perception of emotion in music. However, research on perception may not fully explain children's ability to express emotions through music. Majority of the perception research uses preexisting musical stimuli (usually created by adults) which does not provide sufficient information about the children's own expression of emotion through music. Some pioneering research on expression exists. In a study by Flowers et al. (1997) 5-9-yearold children were asked to create fast, slow, loud, and soft expressions by modifying musical pieces using a synthesizer keyboard. The children were able to manipulate both tempo and dynamics in order to create these contrasts, and they consistently paired slower (longer durations) with softer (quieter) performances even when both conditions 
were not simultaneously required. Adachi and Trehub (1998) asked 4-12-year-old children to express sadness and happiness when singing a song. Already the 4-5-yearolds were able to manipulate tempo, dynamics, and pitch to differentiate happy and sad expression in their singing, and also used a lot of gestures and words. In addition, it has been shown that 4-5-year-olds are able to decode musically expressed sadness and happiness into expressive movement (Boone \& Cunningham, 2001). Overall, however, the knowledge on differences based on age and on the use of particular musical parameters is sparse.

\subsection{Other than age-related individual differences}

As a final note, it can be said that only little is known about the role of individual factors such as gender or musical background. Some studies suggest that expressive skills in music can be taught and learnt (Juslin \& Laukka 2000; Woody, 2000), and Adachi and Trehub (1998) reported that the magnitude of using tempo, pitch, and dynamics to differentiate sadness from happiness differed as a function of children's singing ability. It has also been proposed that boys better encode happiness and anger than girls (Boone \& Cunningham, 2001), but, overall, the knowledge regarding the role of individual background factors in explaining differences in the musical expression of emotion remains inconclusive.

\section{Objectives}

Since less prior knowledge exists on children's expression than perception of emotion in music, the aim of the current study was to particularly investigate how young children use different musical parameters to express basic emotions. Comparative data from 3-year-olds and 5-year-olds was collected in order to investigate age differences a younger sample than what has already been researched in a prior comparative study 
(e.g. Adachi \& Trehub, 1998). We also wanted to go beyond the established finding that children identify the happy-sad dichotomy in music at a very young age. Anger was thus added to the design as a representative of a more complex, yet a possible emotion for a 3-year-old to conceptualize. Regarding musical parameters, we chose to focus on tempo, pitch, and loudness. This was because prior research has already shown that the culturally-based knowledge of using mode as an expression of emotion develops later in childhood (Dalla Bella et al., 2001; Gregory et al., 1996), so we aimed not to repeat that finding but rather focused on providing detailed knowledge about the use of the psychophysiological features in early childhood. The task was therefore to manipulate tempo, pitch, and loudness in order to express the emotions of happiness, sadness, and anger. A novel interface called the Music Box (Bresin \& Friberg, 2011) was used to provide an age-appropriate method for young children to modify the musical material.

Since the chosen musical parameters reflected universal psychophysiological rather than culturally learned emotional expression (Balkwill \& Thompson, 1999; Schubert \& McPherson, 2006; Trehub et al. 2010), and since the chosen emotions were expected to generally be available for conceptualization and expression already at the age of 3 (Lewis, 2000; Saarni, 1999) we hypothesized that both 3-year-olds and 5-yearolds would be able to use all of these parameters in their expression for distinguishing between happiness, sadness and anger. Based on prior research about how these emotions typically are conveyed in musical expression (Gabrielsson \& Lindström, 2010; Juslin \& Timmers, 2010; Juslin \& Laukka, 2004) we expected that it would be possible for the children to use each parameter (tempo, pitch, loudness) to differentiate between sadness and happiness, and also to differentiate between sadness and anger. Specifically, we expected tempo to be higher for happiness and anger than for sadness; pitch to be higher for happiness and anger than for sadness, and loudness to be higher for happiness and anger than for sadness. Based on prior research on these three 
parameters no clear differences between happiness and anger were hypothesized. Gender and musical background were explored to provide overview of the sample, but they were not addressed as variables with hypothesized connections to expression outcomes.

\section{Method}

\subsection{Participants}

The sample consisted of 37 children, a group of 3-year-olds $(n=18 ; 8$ female, 10 male; mean age: 3.7 years) and a group of 5-year-olds $(n=19 ; 10$ female; 9 male; mean age: 5.2 years). The participants were recruited from local daycare centers of the city of Jyväskylä, Finland, and selected only based on age and voluntariness. Consents for the experiment were received from the daycare center directors and the children's parents. Information about the participants' demographic variables and music training was collected through a questionnaire filled in by the parents. Approximately half of the children had participated in music playschool while half had not (3-year-olds: 10 with music playschool experience, 8 without music playschool experience, 5 year olds: 8 with music playschool experience, 11 without music playschool experience).

\subsection{Musical material}

The musical material consisted of excerpts of three children's songs: "Mary Had a Little Lamb", a nursery rhyme from the nineteenth century; "Chim Chim Cher-ee", a song from the musical motion picture Mary Poppins; and a piece called "Hal-mystery", a Halloween song. Three songs were considered to be a suitable amount of songs to modify, in order to have enough variety in the material, and yet not make the study too long for the children. The songs were chosen to represent a differing range of affective 
and musical content and be suitable for the age group. The duration of the excerpts was approximately 30 seconds, after which they were automatically repeated again, resulting in a continuous stimulus that the participants could render as long as they wanted. All pieces were instrumental versions played through the French Horn sound from the Vienna Symphonic Library sound library, and consisting only of the melody line with no lyrics or accompaniment. The order of presenting the songs was randomized for all participants.

\subsection{Music modification interface}

The musical parameters of the songs were modified through a novel interface called the Music Box, which includes separate sliders for each parameter and enables modification of the musical material in real time (Bresin \& Friberg, 2011). The interface is designed to be age-appropriate and easy to use for small children. The slider movements were converted into numerical form with Pure Data software, and the scales for the parameters were: $0-5$ for tempo (slow-fast), $-24-24$ for pitch (low-high), and -10-10 for loudness (quiet-loud). The absolute values of these were not registered, because the study focused on the relative comparison between the expressions.

\subsection{Procedure}

The experiments were conducted in a separate room within the facilities of the day care centers. Each child participated in the experiment alone. At the beginning of the experiment there was a short familiarization phase, during which each participant had the opportunity to try out the sliders and see how they affect the sound. After the familiarization, the participants were asked to use the sliders to make each of the three musical pieces as happy as possible, as sad as possible, and as angry as possible. In order to ensure that all children understood what kind of emotional expression they 
were asked to create, photographs (from Ekman, 1999) representing facial expressions of the target emotions were displayed with the text happy, sad, and angry written in Finnish below the face. The use of facial expressions has been found helpful in research with young children (e.g., Dalla Bella et al., 2001). The participants could render each excerpt as long as they wanted, after which the numeric representations of the slider positions were saved as the measure of the participants' opinion for optimal expression. The duration of each experimental session was approximately 15-20 minutes.

\subsection{Statistical analyses}

Repeated-measures ANOVAs were separately calculated for each of the musical parameters (tempo, pitch, loudness) to investigate the main effect of emotion (happy, sad, and angry expressions). Bonferroni correction was applied to the pairwise comparison between emotions. The main effect of song and interaction between song and emotion was additionally investigated to check that the results would not differ based on the song context (the three different songs). Differences between 3-year-olds and 5-year-olds were investigated by entering age as a between-subject factor in the ANOVAs. As an additional exploration, gender and music playschool experience were also entered as between-subject factors. SPSS statistics Version 22 was used for all analyses.

\section{Results}

Table 1 lists the mean scores for tempo, pitch and loudness in happy, sad, and angry expressions. The scores are averaged across the songs, because there were no significant main effects of song for any parameter, nor were there any interactions between emotion and song for any parameter. 
Significant main effect of emotion was found for all musical parameters, for tempo, $(\mathrm{F}(2,35)=23.11 ; \mathrm{p}<0.01$, pitch $(\mathrm{F}(2,35)=17.02 ; \mathrm{p}<0.01)$, and loudness $(\mathrm{F}(2,35)$ $=15.56 \mathrm{p}<0.01)$.

- Table 1 here -

In line with the hypotheses, tempo was high in happy and angry expressions and low in sadness. Pairwise comparisons showed that sad expression differed significantly from both happy (mean difference $-1.20 ; \mathrm{p}<.001$ ) and angry (mean difference $-1.25 ; \mathrm{p}<$ .001) expressions, while there was no significant between happiness and anger (mean difference $0.05 ; \mathrm{p}=$ n.s.). As hypothesized, there was a similar pattern for pitch: happiness and anger were both expressed with high pitch with no significant difference between them (mean difference $3.07 ; \mathrm{p}=$ n.s.), while sadness was characterized by the lowest pitch values that differed significantly from both happy (mean difference 15.27; $\mathrm{p}<.001$ ) and angry (mean difference 10.28; $\mathrm{p}<0.01$ ) expressions. For loudness, the results were almost in line with the hypothesis. Loudness was highest in anger and lowest in sadness and pairwise comparisons revealed differences in loudness between all expressions: between angry and happy (mean difference $3.54 ; \mathrm{p}<0.01$ ), angry and sad (mean difference 6.32; $\mathrm{p}<0.001$ ), and an almost significant difference between happy and sad (mean difference $2.78 ; \mathrm{p}=0.065$ ).

Age comparison showed little difference between the expressions of 3-year-olds and 5year-olds, confirming our expectation about 3-year-olds already being equally competent than 5-year-olds in using the chosen parameters in differentiating between the emotions. The mean scores for tempo, pitch, and loudness in happy, sad, and angry expressions of 3-year-olds and 5-year-olds are illustrated in Figure 1. No significant 
differences were found, except for an interaction effect of age group and emotion for loudness $(F(2)=3.86 ; p<.05)$, with 3 -year-olds expressing anger with a louder volume than 5-year-olds.

- $\quad$ Figure 1 here -

While no hypotheses were presented for other individual variables than age, we additionally explored whether gender and music background would impact the expressions. No gender differences were observed, but a significant interaction effect of music playschool experience and emotion was observed for pitch $(F(2)=6.46$; $p<$ $.01)$ : children with music playschool training expressed anger with significantly lower pitch values than children with no training (Figure 2). Furthermore, a significant interaction $(F(1)=5.60 ; p<.05)$ for playschool and age for pitch was observed: playschool training was related to the lower pitch for anger expression only in 5-yearolds, not in 3-year-olds. No interactions of age and playschool for tempo and loudness were found.

- $\quad$ Figure 2 here -

\section{Discussion}

The children participating in the study showed great ability in expressing emotional content through music: main effect of emotion was found for all of the studied musical features, and the observed differences between emotions were generally in line with the hypotheses. The overall observation about young children's capacity in expressing basic emotions confirms previous notions about the early ability for emotion expression in music (Adachi and Trehub 1998; Hallam, 2006; Schubert \& McPherson 2006; Trehub, 
2001; Trehub et al. 2010), and for emotion expression in general (Lewis, 2000). Even if the receptive abilities of the children in identifying emotions in music show some differences from those of adults (e.g., Dalla Bella, Peretz, Rousseau, \& Gosselin, 2001; Nawrot, 2003; Nieminen, Istok, Brattico, \& Tervaniemi, 2012; Stachó, 2013; Terwogt \& van Grinsven, 1991), the ways that the children in the current study used the particular musical parameters in the expressions of emotions was exactly in line with how these parameters have been found to be used in the expression of musical emotions in adults (e.g., Gabrielsson \& Lindström, 2001; 2010; Juslin \& Laukka, 2003; 2004; Juslin \& Timmers, 2010). Sadness expression was significantly differentiated from happiness and anger expression, particularly using tempo and pitch. For the case of loudness, the difference between sadness and anger was clear but the difference between sadness and happiness only approached significance: this is, however, not that surprising because happiness also in adults is associated with only a medium-high to high sound level in comparison to high sound level of anger expression (Gabrielsson \& Lindström, 2010; Juslin \& Timmers, 2010; Juslin \& Laukka, 2004).

A core finding of the study was the lack of differences between the 3-year-olds and 5-year-olds. The results establish 3-year-olds as equally competent as 5-year-olds in using the musical parameters of tempo, pitch and loudness in differentiating between the emotional expressions for sadness, happiness, and anger. Concerning general emotional development, anger modulation is still somewhat undeveloped in 3-year-olds, while this ability already is established in 5-year-olds (Watson \& Bell, 2013). Prior research in music has also confirmed that there is early ability for differentiating between sadness and happiness, yet remained somewhat inconclusive about young children's precision in musical anger expression (e.g., Boone \& Cunningham, 2001; Thompson, Schellenberg, \& Husain, 2004; Robazza et al., 1994; Terwogt \& van Grinsven, 1991). The current findings confirm that the differentiation of sadness and 
anger - not only sadness and happiness - is fluent in the musical expression of 3-yearolds.

Concerning the additional exploration on potential differences based on gender and music background, the results showed one difference: children who had experience in music playschool expressed anger with significantly lower pitch values than children without experience in music playschool, and this effect of playschool was only present in 5-year-olds, not in 3-year-olds. 5-year-olds are likely to have engaged in playschool activities longer than the three years olds, and perhaps the result therefore reflects something about how they have learned to express anger musically, while the threeyear-olds might be thinking more purely intuitively. However, with a relatively small sample size and the study not specifically designed to measure the impact of music education (we did not ask for details about the amount and type of the music playschool), these results must be interpreted with great caution. Nonetheless, the finding does point towards a need for further research about the potential impact of early music education on children's development of musical, and possibly also their general, emotional expression.

Some limitations of the study must be mentioned. The sample was relatively small and restricted to western context. A clear limitation is that the setting was restricted to the three parameters of tempo, pitch, and loudness. Mode was purposefully left out of the scope of the study, because the focus was directed on elaborating on the psychophysiological features, but future research could benefit from adding features that are dependent on cultural learning such as mode, or other psychophysiological and expressive features, such as timbre, to the design. The setting was also limited to the three emotions of happiness, sadness and anger, with happiness and anger showing little differences between each other in tempo, pitch, and loudness also in adults (Gabrielsson \& Lindström, 2001; 2010; Juslin \& Laukka, 2003; 2004; Juslin \& Timmers, 2010). 
Future research could benefit in adding other emotions such as tender and fear into play. Stimulus material did not seem to impact the results (no differences in use of musical parameters between songs; no interactions between song and emotion). However, this is partly explained by the stimulus material of the current study, which generally was a relatively homogeneous set of children's songs played with the same timbre. Research on possible interactions in how certain parameters link to certain emotions in different types of musical material would require a design that particularly targets differences in the stimulus material such as contrasting musical genres, use of non-typical musical passages (Webster \& Weir, 2005), or particular combinations of musical features and emotions (Behrens \& Green, 1993). In future work, songs could be purposefully selected and pre-tested to represent particular affective qualities to test whether the song qualities impact the way that parameters are used for expressing certain emotions. Finally, the study focused purely on the musical expression as an outcome, discarding the process of generating it. An interesting topic for future research would be, for instance, to measure the duration for manipulating the three parameters as an indication of the task difficulty. As a process, manipulating three sliders at a same time may be more challenging for a 3-year-old than for a 5-year-old, so the interaction of age and working speed could be tested.

Methodologically, the current study introduced a novel setting for measuring the use of particular musical parameters in the expression of particular emotions. Musical factors do not work in isolation (Gabrielsson \& Lindström, 2001) and therefore it is not optimal for the ecological validity of music research to make observations using one musical factor at a time. The interface Music Box (Bresin \& Friberg, 2011) worked well with this age group and allowed simultaneous real time modification of several musical parameters. This study is one of the few experiments allowing children to act as active contributors to music - a different role from the perceptive and receptive roles that have 
been typical for laboratory experiments in these age groups. This method and way of analyzing children's active contribution to the emotional information carried in the musical parameters can shed light into the roles of different parameters in conveying emotions, and in the future, potentially many other aspects of expression such as social intentions (e.g. domineering, conciliatory) or other meaningful content (e.g. preference and liking, playfulness and humor) in children's musical expression.

Overall, the current study demonstrated that children's capacity of using music to express emotions is in line with the notions of general emotion research about 3-yearolds already being highly capable in emotional expression (Lewis, 2000; Saarni, 1999). The early use of the fundamental, physiological features of loudness, tempo, and pitch has been theorized and discussed in music research, but the current study empirically established that these features indeed allow children as young as 3-year-olds to efficiently convey happiness, sadness, and also anger. The current findings provide background knowledge for understanding the normative development in musical expression of emotion. Knowledge about what musical parameters children can be expected to master for expressing particular emotions can inform both music education practices for learning musical expression or music therapeutic practices dealing with difficulties in emotional development. 


\section{References}

Adachi, M., \& Trehub, S.E. (1998). Children's expression of emotion in song. Psychology of Music, 26(2), 133-153.

Behrens, G.A \& Green, S.B. (1993). The ability to identify emotional content of solo improvisations performed vocally and on three different instruments. Psychology of Music, 21, 20-33.

Bigand, E., Vieillard, S., Madurell, F., Marozeau, J., \& Dacquet, A. (2006). Multidimensional scaling of emotional responses to music: the effect of musical expertise and of the duration of the excerpts. Cognition and Emotion, 19, 11131139.

Boone, R. \& Cunningham, J.G. (2001). Children's expression of emotional meaning in music through expressive body movement. Journal of Nonverbal Behavior, 25, $21-41$.

Bresin, R. \& Friberg, A. (2011). Emotion rendering in music: Range and characteristic values of seven musical variables. Cortex, 47(9), 1068-1081

Cunningham, J. G. \& Sterling, R. S. (1988). Developmental change in the understanding of affective meaning in music. Motivation and Emotion, 12 (4), $399-413$.

Dalla Bella, S., Peretz, I., Rousseau, L., \& Gosselin, N. (2001). A developmental study of the affective value of tempo and mode in music. Cognition, 80(3), B1-B10.

Dolgin, K. G., and Adelson, E. H. (1990). Age changes in the ability to interpret affect in sung and instrumentally-presented melodies. Psychology of Music 18, 87-98.

Eerola, T., Friberg, A., Bresin, R. (2013). Emotional expression in music: contribution, linearity, and additivity of primary musical cues. Frontiers in Psychology, 4, 487. 
Ekman, P. (1999). Basic Emotions. Handbook of Cognition and Emotion, (eds. T. Dalgleish \& M. Power), 45-61. John Wiley \& Sons Ltd, England.

Flowers, P.J. (1997). Structured and Unstructured Musical Contexts and Children's Ability to Demonstrate Tempo and Dynamic Contrasts. Journal of Research in Music Education, 45(3), 341-355.

Fredrickson, W. E. (2000). Perception of tension in music: musicians versus nonmusicians. Journal of Music Therapy, 37, 40-50.

Gabrielsson, A. \& Lindström, E. (2001). The influence of musical structure on emotional expression. Music and emotion: theory and research, (eds. P. N. Juslin \& J. A. Sloboda), 223-248. Oxford University Press, New York.

Gabrielsson, A. \& Lindström, E. (2010). The role of structure in the musical expression of emotions. In Juslin PN and Sloboda J (Eds), Handbook of Music and Emotion: Theory, Research, Applications, pp. 367-400. Oxford University Press, New York.

Gerardi, G. M. \& Gerken, L. (1995). The Development of Affective Responses to Modality and Melodic Contour. Music Perception, 12 (3), 279-290.

Gregory, A. H., Worrall, L., \& Sarge, A. (1996). The development of emotional responses to music in young children. Motivation and Emotion, 20, 341-348.

Hallam, S. (2006). Musicality. The child as musician: The handbook of musical development, (ed. G, E. McPherson), 93-111. Oxford University Press, New York. Juslin, P.N. (1997). Emotional communication in music performance: A functionalist perspective and some data. Music Perception, 14, 383-418.

Juslin, P.N. \& Laukka, P. (2000). Improving emotional communication in music performance through cognitive feedback. Musicae Scientiae, 4(2), 151-183 
Juslin, P. N., \& Laukka, P. (2003). Communication of emotions in vocal expression and music performance: Different channels, same code? Psychological Bulletin, 129, $770-814$.

Juslin, P.N., \& Laukka, P. (2004). Expression, perception, and induction of musical emotions: a review and a questionnaire study of everyday listening. Journal of New Music Research, 33(3), 217-238

Juslin, P.N. \& Timmers, R. (2010). Expression and communication of emotion in music. In P.N. Juslin, J. Sloboda (Eds.), Handbook of music and emotion: theory, research, applications, Oxford University Press (2010), pp. 453-92.

Kastner, M.P. \& Crowder, R. G. (1990). Perception of the major/minor distinction: IV. Emotional connotations in young children. Music Perception, 8, 189-202.

Krumhansl, C.L. (2000). Rhythm and Pitch in Music Cognition. Psychological Bulletin, 126(1), 159-179.

Krumhansl, C.L. \& Keil, F.C. (1982) Acquisition of the hierarchy of tonal functions in music. Memory \& Cognition, 10, 243-251

Lewis, M. (2000). The emergence of human emotions. Handbook of Emotions, (eds. M. Lewis \& J.M. Haviland-Jones), 265-280. The Guilford Press, New York.

Nawrot, E.S. (2003). The perception of emotional expression in music: evidence from infants, children and adults. Psychology of music, 31, 75-92.

Nieminen, S., Istok, E., Brattico, E., \& Tervaniemi, M. (2012). The development of the aesthetic experience of music: preference, emotions, and beauty. Musicae Scientiae, 16 (3), 372-391.

Peretz, I., Gagnon, L., \& Bouchard, B. (1998). Music and emotion: Perceptual determinants, immediacy, and isolation after brain damage. Cognition, 68, 111141. 
Perlman, M., Krumhansl, C.L. (1996). An experimental study of interval standards in Javanese and Western musicians. Music Perception, 14(2), 95-116

Robazza, C., MacAluso, C., \& D’Urso, V. (1994). Emotional reactions to music by gender, age, and expertise, Perceptual and Motor Skills, 79 (2), 939-44

Russell, J. A. (1980). A circumplex model of affect. Journal of Personality and Social Psychology. 1980; 39, 1161-1178.

Schubert, E., McPherson, G.E. (2006). The perception of emotion in music. The child as musician: The handbook of musical development, (ed. G, E. McPherson), 193212. Oxford University Press, New York.

Saarni, C. (1999). The development of emotional competence. Guilford press: New York.

Shuter-Dyson \& Gabriel 1981. The psychology of musical ability. Routledge Kegan \& Paul.

Stachó, L., Saarikallio, S., Huotilainen, M., vanZijl, A., \& Toiviainen, P. (2013). Perception of emotional content in musical performances by 3-7-year-old children. Musicae Scientiae, 17 (4), 495 - 512.

Terwogt, M. M., \& van Grinsven, F. (1991). Musical expression of moodstates. Psychology of Music, 19, 99-109.

Thompson, W. F. Schellenberg, E. G. \& Husain, G. (2004). Decoding speech prosody: do music lessons help? Emotion, 4(1), 46-64.

Trehub, S.E. (2001). Musical predispositions in infancy. Annals of the New York Academy Sciences, 930, 1-16.

Trehub, S.E., Hannon, E.E., \& Schachner, A. (2010). Perspectives on music and affect in the early years. In P.N. Juslin, J. Sloboda (Eds.), Handbook of Music and Emotion: Theory, Research, Applications, Oxford University Press (2010), pp. 645-68. 
Vieillard, S., Peretz, I., Gosselin, N., Khalfa, S., Gagnon, L., \& Bouchard, B. (2008). Happy, sad, scary, and peaceful musical excerpts for research on emotions. Cognition \& Emotion, 22, 720-52.

Watson, A. I. \& Bell, M. A. (2013) Individual Differences in Inhibitory Control Skills at Three Years of Age. Dev Neuropsychol. 2013; 38(1): 1-21.

Webster, G. D., \& Weir, C. G. (2005). Emotional responses to music: Interactive effects of mode, texture, and tempo. Motivation and Emotion, 29, 19-39.

Woody, R.H. (2000). Learning expressivity in music performance. Research Studies in Music Education, 14(1), 14-23. 
Table 1. Mean scores and standard deviations for tempo, pitch, and loudness in happy, sad and angry expressions, averaged across songs.

\begin{tabular}{llllllll}
\hline Parameter & Scale & Happy & \multicolumn{3}{c}{ Sad } & \multicolumn{3}{c}{ Angry } \\
\hline \multirow{2}{*}{ Tempo } & $0-5$ & Mean & SD & Mean & SD & Mean & SD \\
Pitch & -2.32 & 0.96 & 1.12 & 0.80 & 2.37 & 1.12 \\
Loudness & $-10-10$ & -0.54 & 5.40 & -3.32 & 4.87 & 2.99 & 4.87 \\
\hline
\end{tabular}



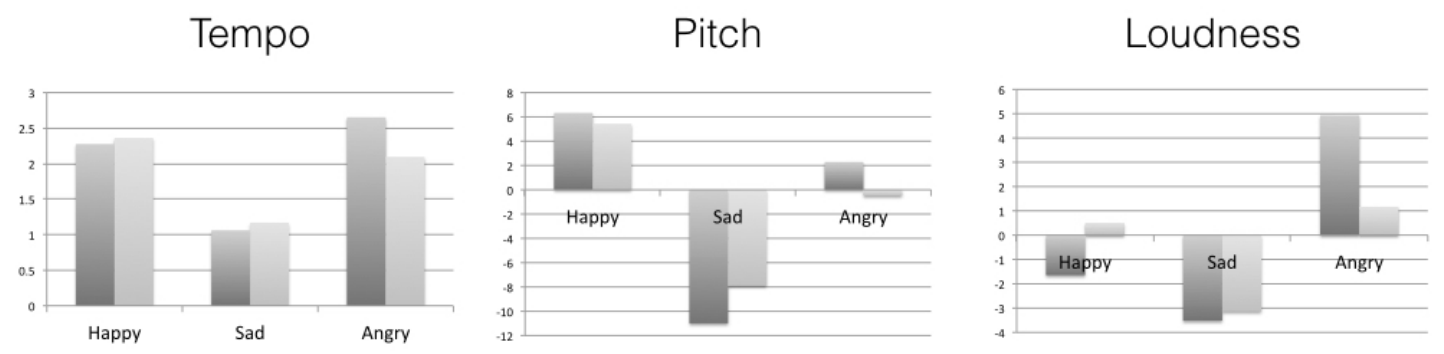

3-year-olds

5-year-olds

Figure 1. Mean scores for tempo, pitch, and loudness for happy, sad, and angry expressions in 3-year-olds and 5-year-olds. 


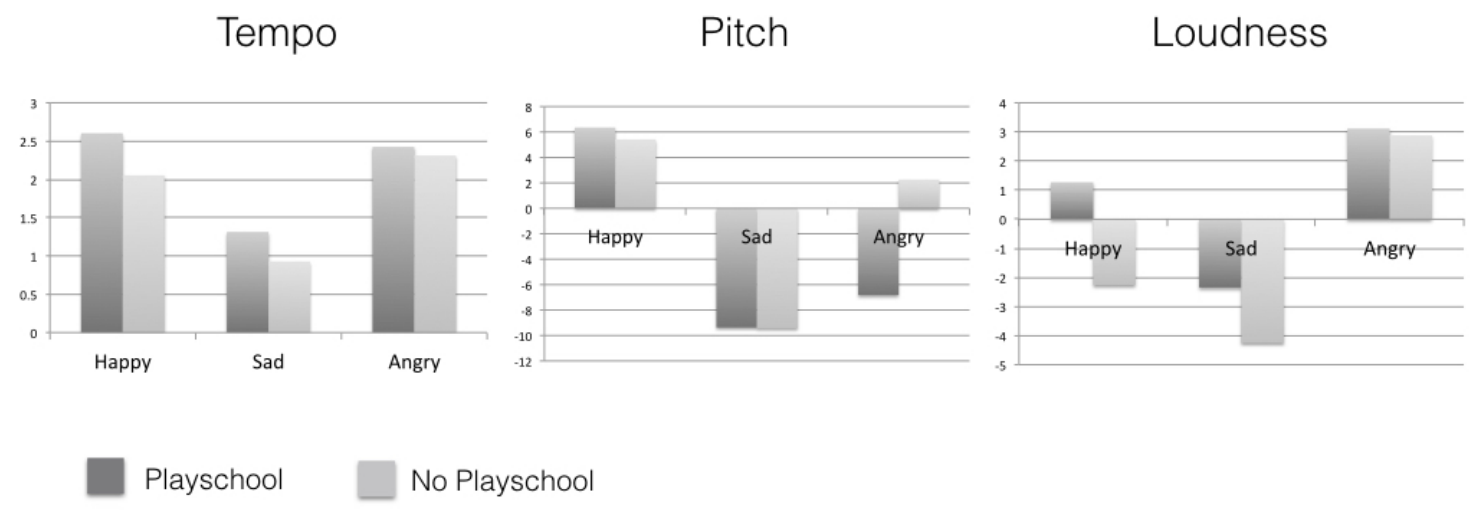

Figure 2. Mean scores for tempo, pitch, and loudness for happy, sad, and angry expressions in children with and without music playschool experience. 